\title{
IFEDC-20194285 Phase-controlled Inversion Under High-resolution Pseudo-impedance Curve
}

\author{
Zhang Ruyue*, Deng Xiaojiang, Wang Xiaolan, Tan Rongbiao, Luo Jing, Guo Yuchen, Li Yangjing, \\ Huang Lijuan, Li He
}

Southwest Geophysical Research Institute of China National Petroleum Corporation, Chengdu, China

\author{
Email address: \\ zhangry_wt@cnpc.com.cn (Zhang Ruyue) \\ ${ }^{*}$ Corresponding author
}

\section{To cite this article:}

Zhang Ruyue, Deng Xiaojiang, Wang Xiaolan, Tan Rongbiao, Luo Jing, Guo Yuchen, Li Yangjing, Huang Lijuan, Li He. IFEDC-20194285 Phase-controlled Inversion Under High-resolution Pseudo-impedance Curve. American Journal of Mechanics and Applications.

Vol. 9, No. 1, 2021, pp. 6-11. doi: 10.11648/j.ajma.20210901.12

Received: November 22, 2020; Accepted: December 25, 2020; Published: February 23, 2021

\begin{abstract}
Currently, conventional seismic inversions are mostly premised on well data, and there exist uncertainty in seismic inversions in areas with few fixed wells. After years of production practice, well-seismic cooperative inversion under the constraint of pseudo wells is a more effective method for reservoir prediction in areas with few fixed wells and complex phase spread changes. Nevertheless, the selection of locations of conventional pseudo wells only takes into account the spatial distribution of wells but seldom considers the changes of phase spreads; curves of pseudo wells are mostly derived from sparse pulse inversion with relatively low longitudinal resolution, which cannot accurately reflect the vertical and horizontal variation characteristics of the reservoir; there is no effective quality control method to prove the reliability of the curve after selecting the pseudo well. To solve the above-mentioned problems, this thesis acquires pseudo-wave impedance curves with high-frequency components by means of waveform difference inversion, controls quality and optimizes the location of pseudo wells under the guidance of geological results, and performs phase control inversion under the constraint of high-frequency pseudo-wave impedance curves, which greatly enhances the vertical and horizontal resolution of inversion, solves the problem of fine reservoir prediction in areas with few fixed wells, and offers strong support for high-speed and efficient development of oil and gas.
\end{abstract}

Keywords: Pseudo Well, High Resolution Ratio, Pseudo Impedance Curves, Phase Control

\section{Introduction}

Traditional reservoir seismic inversions are a special technology that predicts the lithology, morphology, physical properties and oil-gas bearing property of oil and gas reservoirs employing seismic information as the main basis and comprehensively regarding geological, logging, petrophysical and other information as constraints. Currently, most inversion techniques are premised on the information of fixed wells, using interwell interpolation or geostatistical methods for modeling, combined with the comprehensive constraint inversion technique characterized by intensive horizontal sampling of seismic data [1]. The dependence of this inversion method on the quantity and quality of well data leads to an increase in uncertainty of inversion results in areas with few wells. Predecessors have solved this problem by participating pseudo wells, but the selection of locations of conventional pseudo wells usually only considers the spatial distribution of wells but is lack of geological rationality [2]. The extracted pseudo curves are mostly derived from sparse pulse inversions, resulting in that the frequency of pseudo wells is equivalent to the seismic frequency and the accuracy of inversion prediction is relatively low.

The method employed in this thesis breaks away from the requirement of the traditional inversion method for the number of wells [3], can break through the limitations of low resolution of traditional sparse pulse inversions and insufficient geostatistical inversion samples in areas with few fixed wells and realize phase-controlled inversion under the constraint of optimal selection of high-resolution pseudo-impedance curves. 


\section{Technical Idea}

(1) The change of seismic waveform reflects the change of sedimentary facies, and the high-frequency pseudo wave impedance curves extracted by waveform difference inversion boasts great "phase control" property;

(2) The existing seismic and geological data are effectively utilized to optimize the locations of pseudo wells, so that the well locations are evenly distributed in different phase spreads, and the curves of pseudo wells are optimized via the sorting of well-seismic correlation;

(3) Inversion is conducted under the constraint of high-frequency pseudo wave impedance curves with phase control characteristics.

\section{Methods and Principles}

\subsection{Waveform Difference Inversion}

\subsubsection{Waveform Difference Theory}

Waveform difference inversion is similar to geostatistical inversion, with intermediate frequency constrained by earthquake and high frequency derived from well data [4]. However, traditional geostatistical inversion acquires parameter values (such as range variation and base station value) that characterize the spatial structure of the reservoir by performing variogram calculation and analysis on the data of fixed well drilling [5]. Cross-well variables are estimated by selecting relevant samples based on the range variation size, and the statistical results of probability are given. This inversion method relies heavily on the original well data, and

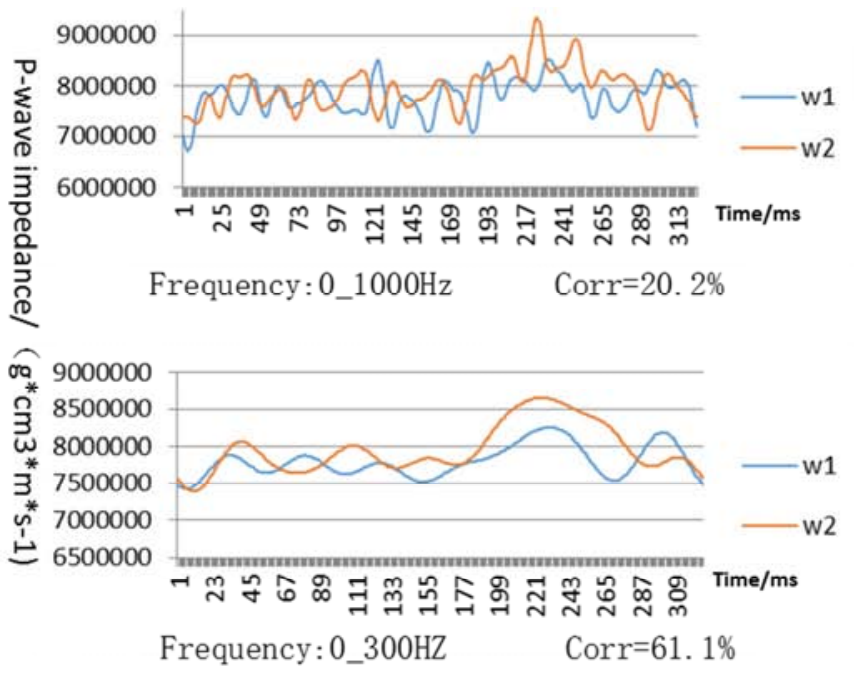

the number of fixed drilling wells and the spatial distribution of well locations directly affect the inversion accuracy. In areas with few wells and fast phase spread change, the errors caused by fewer wells and uneven distribution of well locations will be greater [6].

In light of previous studies, seismic waveform represents the tuning pattern of vertical lithologic combination of reservoir, and the change of its transverse waveform reflects the phase change characteristics of reservoir space, is associated with sedimentary environment, and can well represent the vertical and horizontal changes of reservoir. Under the assumption of wavelet invariance, it is considered that the difference of seismic impedance is closely associated with the change of well impedance structure, that is, the change of seismic waveform originates from the change of well impedance [7]. The low frequency part of the wave impedance curves represents its sedimentary environment, if the seismic waveforms are similar, the low-frequency wave impedance curves of the seismic traces will be similar, indicating that they are in the same phase spread.

By comparing the wave impedance curves of two wells in the same phase spread (Figure 1), it can be fixed that the similarity of the curves is associated with the frequency bandwidth, from high frequency to low frequency, from completely random to gradually determined. In the simulation process, the frequency component of the seismic trace to be predicted is also a process from low to high, from determination to step-by-step determination and then to random. The certainty of the inversion higher than the seismic frequency part is greatly enhanced compared with the random simulation of traditional geostatistics.

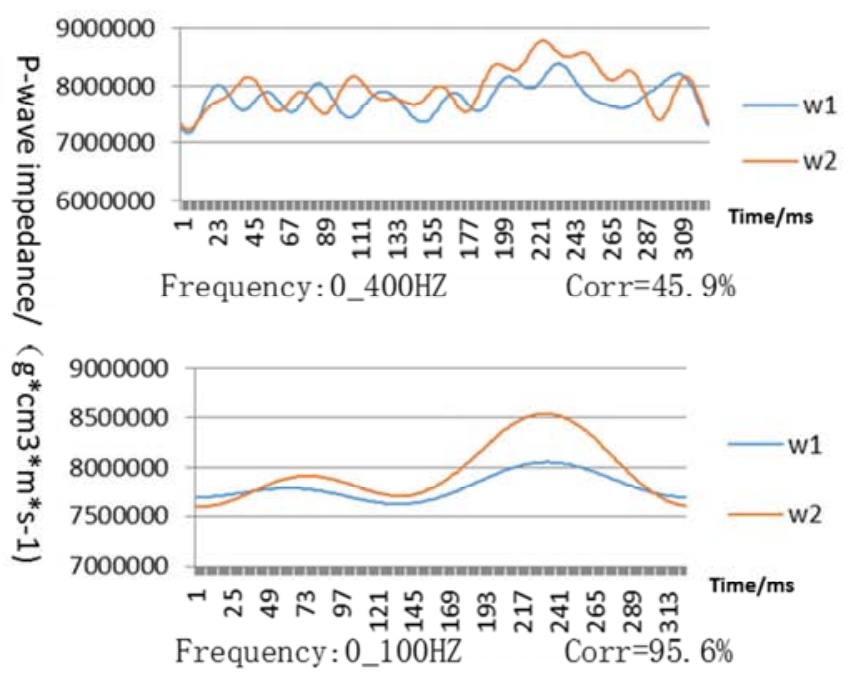

Figure 1. Relationship between Seismic Waveform and Wave Impedance.

Waveform difference inversion makes full use of seismic data with dense spatial distribution, selects statistical samples based on the impedance difference of seismic traces, that is, the similarity of impedance residual traces, and conducts random simulation to establish an initial model. Using waveform difference inversion algorithm for phase-controlled impedance inversion and extracting high-frequency pseudo-impedance curves to participate in the next inversion are the core of phase-controlled inversion under the constraint of optimal selection of high-resolution pseudo-impedance curves.

\subsubsection{Feature Vectors of Waveform Difference}

The following four feature vectors can be employed to 
describe the difference in seismic impedance

(1) Center of gravity: reflecting the geometric relationship between the amplitude of each sampling point in the same time window and the same time window on the time plane.

$$
\mathrm{RM}=\frac{\sum_{i=1}^{N} i * x(i)}{N * \sum_{i=1}^{N} x(I)}
$$

$\mathrm{x}(\mathrm{i})$ is the amplitude value corresponding to Point $\mathrm{i}$, and $\mathrm{N}$ is the number of sampling points in the time window

(2) Mean value: the mean value of the seismic amplitude of each sampling point in the same time window, which roughly depicts the average amplitude in the same time window

$$
\mathrm{X}=\sum_{i=1}^{N} \frac{x(i)}{N}
$$

$\mathrm{x}(\mathrm{i})$ is the amplitude value corresponding to Point $\mathrm{i}$, and $\mathrm{N}$ is the number of sampling points in the time window

(3) Variance: describing the degree to which the amplitude of all sampling points in the same time window deviates from its mean value

$$
\mathrm{S}=\frac{1}{N-1} \sum_{i=1}^{N}[x(i)-x]^{2}
$$

$\mathrm{x}(\mathrm{i})$ is the amplitude value corresponding to Point $\mathrm{i}$, and $\mathrm{N}$ is the number of sampling points in the time window

(4) Variable variance: the mean value of the sum of squares of the amplitude differences between two sampling points with interval $\mathrm{h}$ in the same time window

$$
\mathrm{r}(\mathrm{h})=\frac{1}{N(h)} \sum_{i=1}^{N}[x(i)-x(i+h)]^{2}
$$

$\mathrm{x}(\mathrm{i})$ is the amplitude value corresponding to Point $\mathrm{i}$, and N(h) is the number of sampling points with interval h-1 in the time window

\subsection{Inversion Process of Waveform Difference}

(1) Acquire impedance residual traces by comparing the wave impedance curves of the well-side seismic traces of two fixed wells, and select similar residual traces by comparing impedance residual traces of the two seismic traces to be predicted with the well-side seismic impedance residual traces of the two fixed wells (Figure 2);

(2) Acquire multiple sampling points by taking the two seismic traces to be predicted as sampling points, starting from the fixed wells and gradually discriminating from near to far to the whole work area; make statistics on the vertical variogram of the above four feature vectors and the fixed well impedance to characterize the "contribution" of the vertical structural change of the well impedance to the seismic impedance difference [8];

(3) Statistically select the feature vectors of the seismic impedance of the sampling points [9], and use the above variogram to simulate the well impedance of the sampling points to acquire the high frequency wave impedance of a sufficient number of sampling points.

(4) In selecting sampling points, to avoid sample selection errors in a large distance range and increase the maximum spatial distance control [10], use the waveform similarity and spatial distance bivariate to select the wells with similar intermediate and low frequency structures as spatial estimation samples in the sampling points to be selected. After low-pass filtering, establish a low-frequency model based on phase-spread changes with

\begin{tabular}{|c|c|c|c|}
\hline & WELL A & WELL B & $\begin{array}{l}\text { difference between } \\
A \text { and } B \text { impedance }\end{array}$ \\
\hline 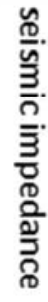 & & & \\
\hline 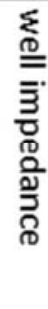 & $\sum$ & 3 & $\sum_{j}^{3}$ \\
\hline
\end{tabular}
the high-frequency impedance of the sampling points and the frame model, and conduct inversion to acquire a high-frequency invertomer as an output.

Figure 2. Comparison of Seismic Impedance and Well Impedance.

In areas where there are few fixed wells, waveform difference inversion employs the relationship between the lateral variation of waveform and the relative variation of well impedance to establish a characteristic vector variogram model [11], which realizes the high-frequency simulation of well-seismic cooperation. The acquired pseudo wave impedance curves boasts high reliability and high vertical and horizontal resolution, offering strong support for subsequent inversion.

\subsection{Extraction of Pseudo Wave Impedance Curve}

Selection of the locations of pseudo wells

To ensure the research effect and better control of the pseudo well on the plane, the selection of well location is of crucial importance. The selection of the pseudo well is premised on the following two principles:

(1) The selection of the locations of pseudo wells should be conducted under the guidance of geological results to meet the sufficient control of well points on different phase spreads;

(2) The quality of seismic data at the pseudo well point is relatively good, avoiding faults [12].

\subsection{Optimization of Pseudo Wave Impedance Curve}

Despite that waveform difference inversion makes up for the limitation of insufficient samples in areas with few wells 
by distinguishing waveform difference and regarding the selected seismic traces as sampling points, and can acquire a high-frequency invertomer in areas with few wells, the inversion result lacks a large amount of well data as a posterior condition for the high-frequency part, so it is necessary to make a selection of the extracted pseudo curve via artificial posterior [13].

The synthetic records acquired by convolution of these pseudo curves and sub-waves are matched with the original seismic traces. Sort these pseudo curves by correlation coefficient, select the pseudo curves with better correlation and the fixed well curves as the original curves for the next inversion, and finally acquire the invertomer with high resolution.

\section{Application of Results}

Block S fault depression in Songliao Basin is a basin controlled by low-angle boundary faults, volcanic rocks are widely developed in the basin, and volcanic rocks of Yingcheng Formation are important reservoirs of deep natural gas in this area. In light of previous studies, the development of volcanic reservoirs in Yingcheng Formation of Block $\mathrm{S}$ is principally controlled by phase spread [14]. The superimposition of multi-phase cycles of volcanic rocks in the area and the rapid change of lateral lithofacies (Figure 3) result in strong vertical and horizontal heterogeneity of volcanic reservoirs, which causes great difficulties to the fine prediction of volcanic reservoirs [15].

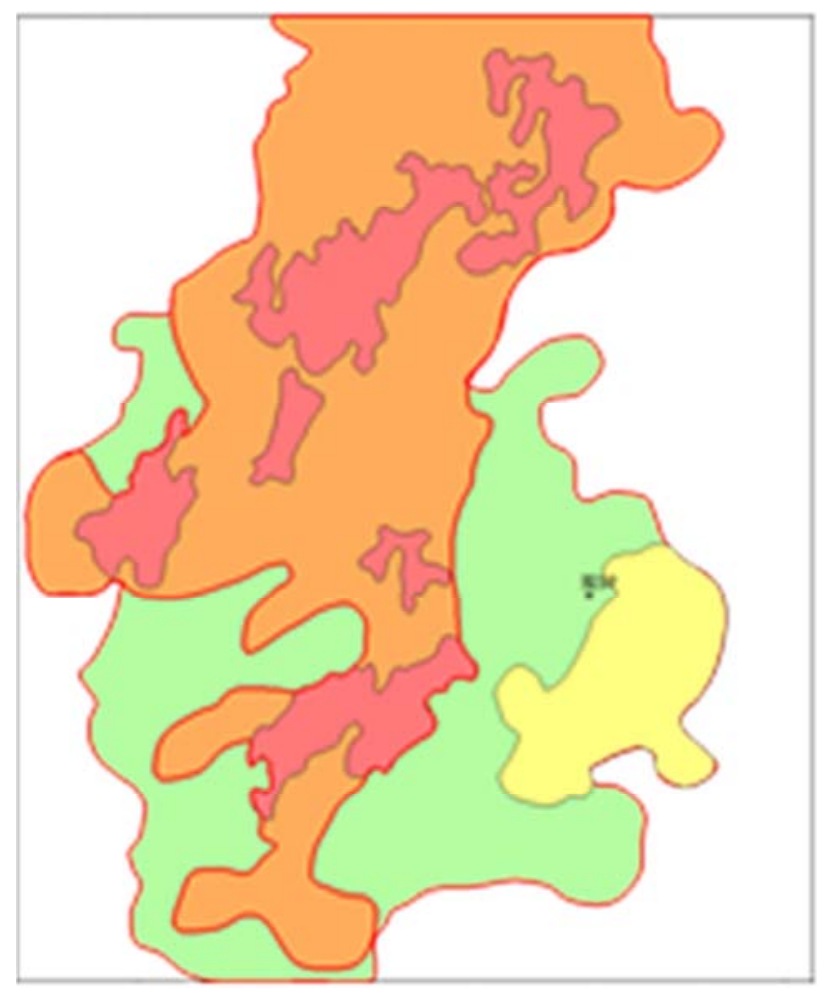

Figure 3. Distribution Diagram of Primary and Secondary Lithofacies of Volcanics in First Section of Yingcheng Formation.

\subsection{Extraction and Selection of Pseudo-well Curves}

Compare the pseudo wave impedance curve of the pseudo well site extracted from the waveform difference invertomer with the pseudo wave impedance curve of the sparse pulse invertomer (Figure 4): the pseudo curve extracted from the sparse pulse invertomer has a frequency band of about $40-50 \mathrm{~Hz}$, which is similar to the seismic frequency band, and is not helpful for improving the longitudinal resolution of subsequent inversion and cannot meet the requirements of fine reservoir prediction. The pseudo curve frequency extracted from the waveform difference invertomer is far beyond the seismic frequency band, reaching $200 \sim 250 \mathrm{~Hz}$, which greatly enhances the longitudinal resolution of subsequent inversion.

Select 10 locations of pseudo wells in which the quality of seismic data is relatively good, avoid faults, and make the well locations evenly distributed in different phase spreads as far as possible (Figure 5); number these pseudo curves and match the synthetic record obtained by convolution of sub-waves with the original seismic trace. The synthetic record of pseudo curve No. 8 in Figure 6a has a higher matching degree with the well-side trace, with a correlation coefficient of 0.94 . The synthetic record of pseudo curve No. 2 in Figure $6 \mathrm{~b}$ at the red arrow has a lower matching degree with the well-side trace, with a correlation coefficient of 0.85 ; sort these pseudo curves by correlation coefficient, and select the first 8 pseudo curves with good correlation as the original curves for the next inversion together with the fixed well curves, and finally acquire the invertomer with high resolution.

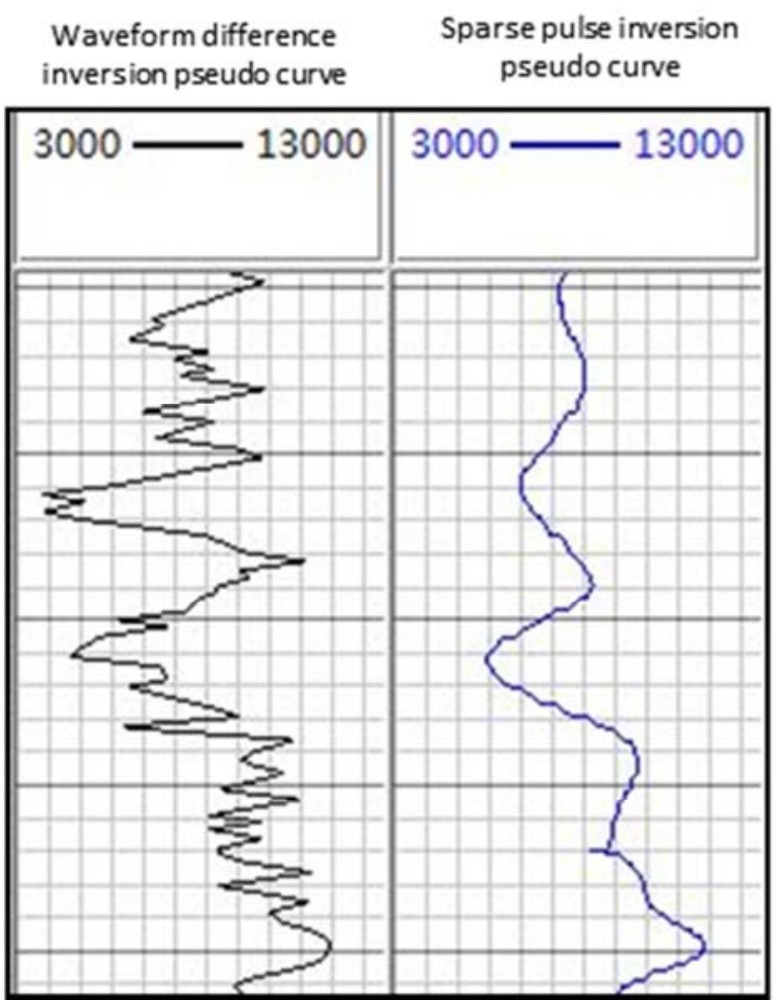

Figure 4. Comparison of Pseudo-Curves between Waveform Indication Inversion and Sparse Pulse Inversion. 


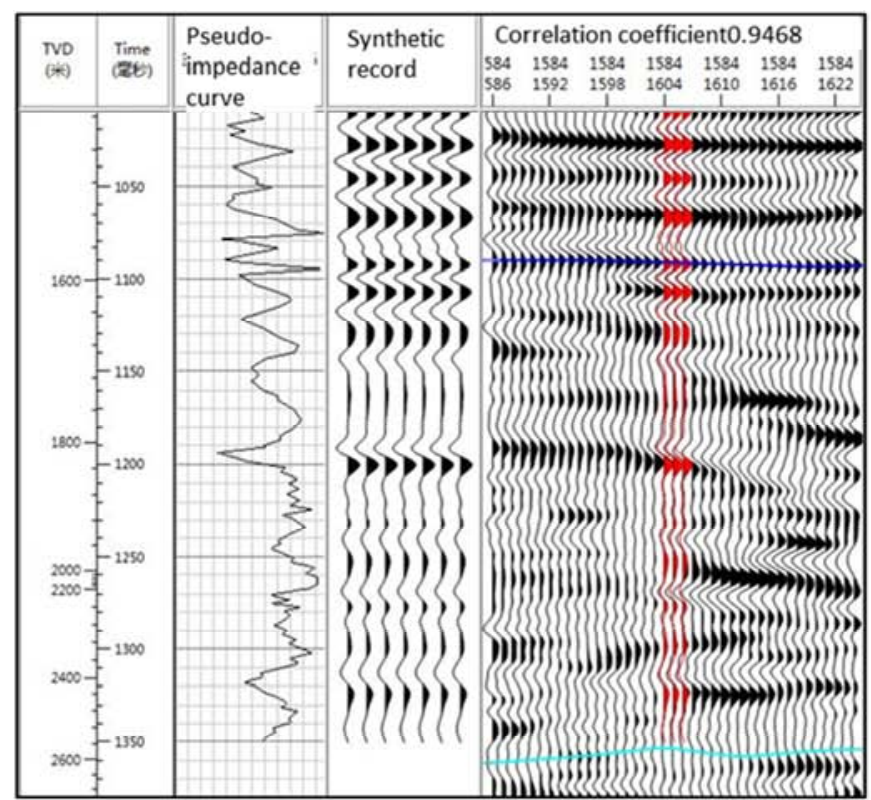

a

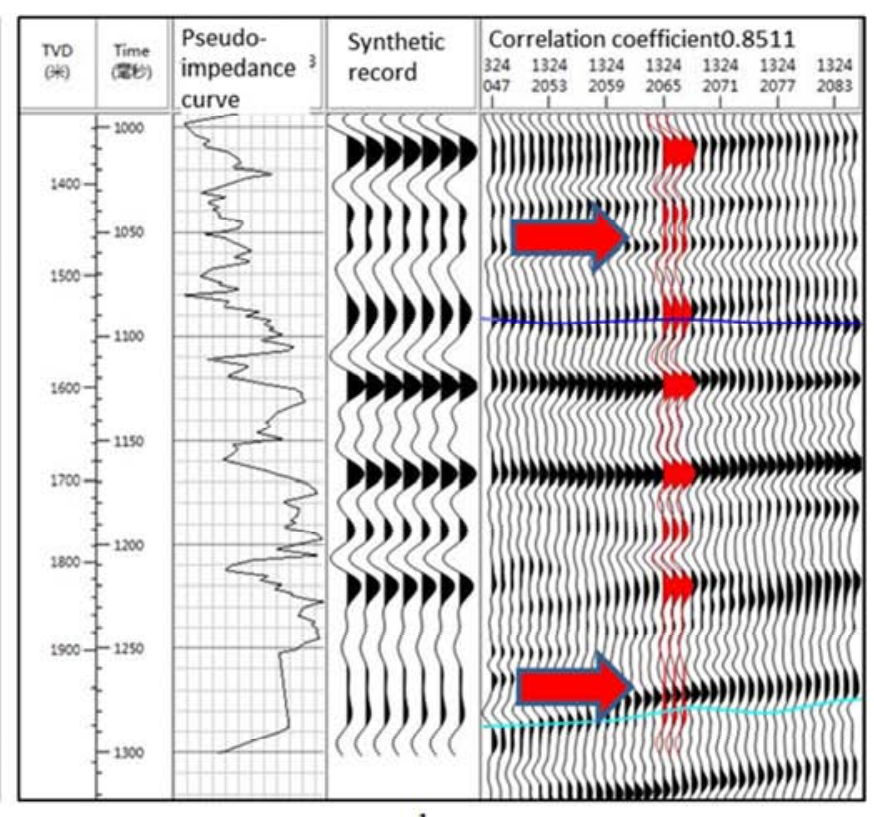

$\mathrm{b}$

Figure 5. a. Well-Seismic Correlation Diagram of No. 8 Pseudo Curve b. Well-Seismic Correlation Diagram of No. 2 Pseudo Curve.

\subsection{Analysis of Inversion Effect}

Figure 6 is a comparison of sparse pulse inversion, waveform difference inversion, and high-resolution pseudo-impedance inversion in $\mathrm{S}$ region. The logging curve is the wave impedance curve, and S11 is the verification well. W8, W5, W6 are preferred pseudo-wells after waveform difference inversion. It can be seen from the profile that the volcanic reservoirs in the area are relatively developed (yellow-red bands).
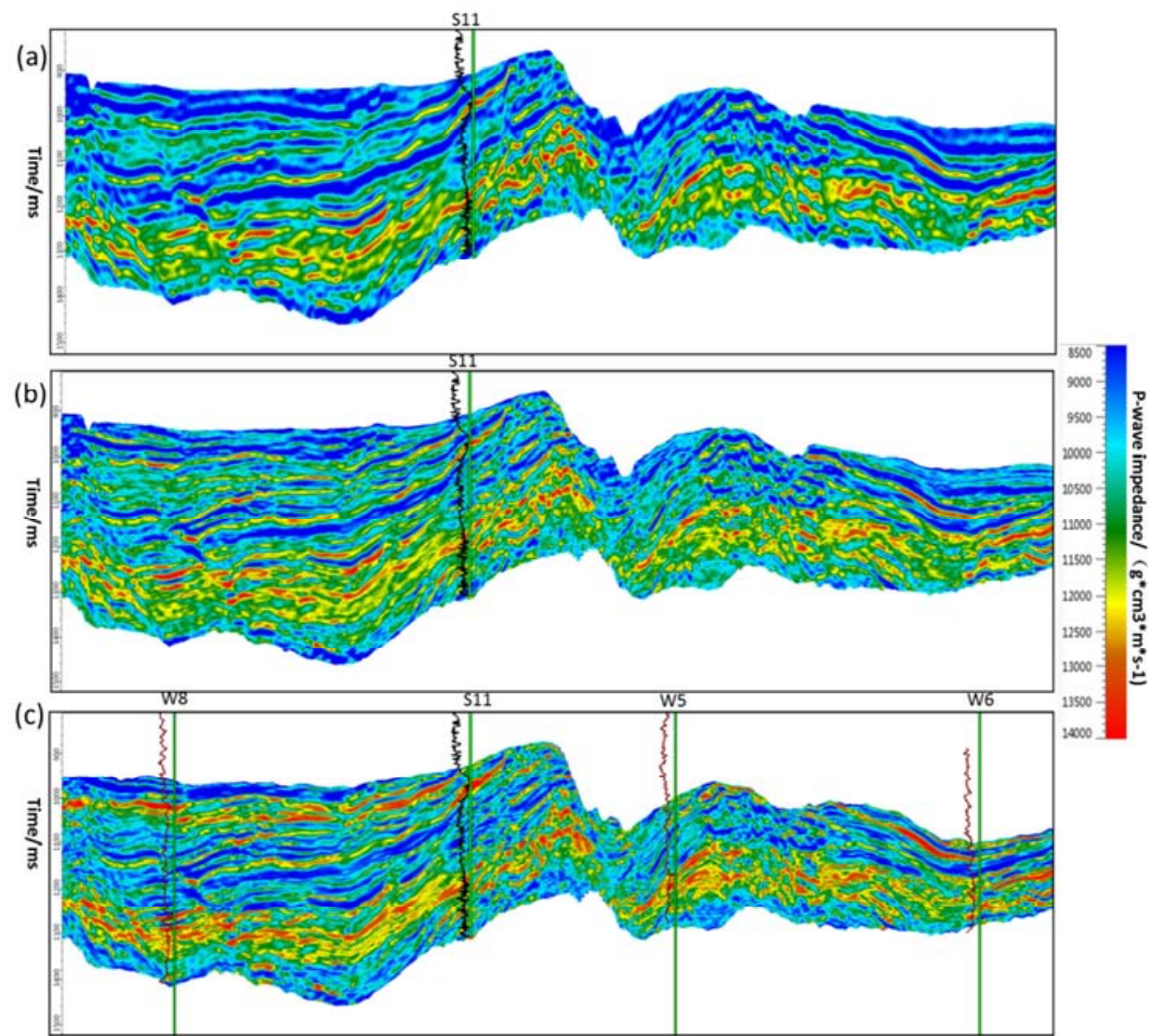

a-Sparse pulse inversion profile; b- Waveform difference inversion profile; c- High resolution pseudo impedance inversion profile

Figure 6. Sparse pulse inversion, waveform difference inversion and high-resolution pseudo-impedance inversion in S region. 
Comparing the results of sparse pulse inversion and waveform difference inversion (profile a and profile b), it can be seen that the lateral distribution of both has the characteristics of seismic facies, and the shape of the crater cone is obvious, However, the vertical resolution of the waveform difference inversion profile is significantly higher than that of the sparse pulse inversion, and the inversion profile at well S11 is in good agreement with the actual well.

Comparing the waveform difference inversion with the high-resolution pseudo-impedance inversion (profile $\mathrm{b}$ and profile c), it can be seen that the vertical resolution of the two is equivalent, but the high-frequency pseudo-impedance inversion adds the control of the pseudo-impedance curve of the virtual well. The reservoir in the upper part of the target layer is better characterized. The lateral distribution of the reservoir is more in line with the multi-period eruption of volcanic rocks, and the inversion profile at well $\mathrm{S} 11$ is more consistent with the actual well.

\section{Conclusions and Recommendations}

(1) Volcanic phase spread in Block S of Songliao Basin changes rapidly, and there are few fixed exploratory wells (two), which brings great difficulties to the fine prediction of reservoir [16]. The pseudo wave impedance curves of high-frequency pseudo well is acquired via waveform difference inversion, which furnishes high-resolution samples with phase control as constraints for subsequent inversion, thereby greatly improving the prediction accuracy.

(2) This inversion breaks through the limitations of low resolution of conventional sparse pulse inversion and insufficient geostatistical inversion samples in areas with few wells, effectively enhances the vertical and horizontal resolution ratio of the prediction results, and comes up with a new idea for high resolution prediction of reservoirs in blocks with few wells and relatively big changes in phase spread.

\section{References}

[1] Rieker N. The form and nature of seismic waves and the structure of seismograms. Geophysies 5 (4), 348-366 (1940).

[2] $\mathrm{Gu}$ wen, $\mathrm{Xu}$ min, Wang duo-han. Waveform indication inversion technique in thin reservoir prediction Application-taking the thin-layer sandstone gas reservoir in the $\mathrm{B}$ area of Junggar Basin as an example. Natural gas geoscience 27 (11), 2064-2069 (2016).

[3] Zhang Xiong, Zhang Benjian, Liang Hong. The application of pre-stack inversion based on seismic waveform indicator to the prediction of compact and thin oil-bearing sand layer. Geophysical and Geochemical Exploration 42 (3), 545-554 (2018).

[4] Yang Guoxiu, Song Liyuan, Shang Jianxia. Application of geostatistical inversion technique in reservoir prediction in the south of Tuo11. Geophysical and geochemical exploration 39 (6), 1193-1198 (2015).

[5] Sheriff R E. Limitations on resolution of seismic reflections and geologic detail derivable from them in seismic stratigraphy-Application to hydrocarbon exploration. AAPG Memoir (26), 3-14 (1977).

[6] Levy S, Fullagar P K. Reconstruction of a sparsespike train from portion of its spectrum and application to high-resolution deconvolution. Geophysics 46 (9), 1235-1243 (1981).

[7] He Huohua, Li Shaohua, Du Jiayuan. Prediction of thin sand body reservoirs using geostatistical inversion. Geophysical and geochemical exploration 35 (6), 804-808 (2011).

[8] Gan Pengzhi, Wang Yuanjun, Ye Yan. Application research of constrained sparse spike inversion in GX gas field. Land and resources technology management 33 (3), 107-113 (2016).

[9] Zhang Zhiming, Zhang Mingxue, Hu Yushuang. Application of prestack inversion to volcano rock reservoir prediction in the area Yingshan-Shuangcheng fault depression. Progressin Geophysics, 30 (2): 0621 - 0627 (2015).

[10] Chuai Y Y, Zhou D H, Ln D Y. Application of prestack simultaneous inversion technique in the hydrocarbon detection of QHD29 2 area in Bohai sea. Journal of Daqing Petroleum Institute, 36 (3): 8-12 (2012).

[11] Dai S L, Jiang C J, Zhang Y G. Methodologies for predicting effective reservoir of volcanic in Anda area of Xujiaweizi depression. Geological Science and Technology Information, 31 (2): 67-71 (2012).

[12] Zheng X D, Li Y D, Yu X W, et al. 2007. Reef and shoal reservoir characterization using paleogeo- morpology constrained seismic attribute analysis [J]. SEG/San Antonio 2007 Annual Meeting, 1382-1386.

[13] Anderson N L, Brown R J, Hinds R C. 1989. Low and high relief Leduc formation reef-A seismic analysis [J]. Geophysics, 54 (11): 1410-1419.

[14] Anderson N L, Franseen E K. 1991. Differential compaction of Winnipegosis reefs: A seismic perspective [J]. Geophysics, 56 (1): $142-147$.

[15] Neves F A, Zahrani M S, Bremkamp S W. 2004. Detection of Potential Fractures and Small Faults Using Seismic Attributes [J]. TLE, 23 (9): 903-906.

[16] Chen Q, Sidney S. 1997. Seismic attribute technology for reservoir forecasting and monitoring $[\mathrm{J}]$. The leading Edge, 16 (5): $220-231$. 